\section{BMJ Open \\ Respiratory \\ Research}

\title{
Associations between genetics, medical status, physical exercise and psychological well-being in adults with cystic fibrosis
}

\author{
Lena Backström-Eriksson, ${ }^{1,2}$ Agneta Bergsten-Brucefors, ${ }^{2}$ Lena Hjelte, ${ }^{2,3}$ \\ Bo Melin, ${ }^{1}$ Kimmo Sorjonen ${ }^{1}$
}

To cite: BackströmEriksson L, BergstenBrucefors A, Hjelte L, et al. Associations between genetics, medical status, physical exercise and psychological well-being in adults with cystic fibrosis. BMJ Open Resp Res 2016;3 e000141. doi:10.1136/ bmjresp-2016-000141

- Additional material is available. To view please visit the journal (http://dx.doi.org/ 10.1136/bmjresp-2016000141)

Received 8 April 2016 Revised 24 August 2016 Accepted 30 August 2016

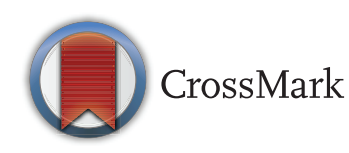

${ }^{1}$ Department of Clinical Neuroscience, Division of Psychology, Karolinska Institutet, Solna, Sweden ${ }^{2}$ Karolinska University Hospital, Stockholm CFcenter, Stockholm, Sweden ${ }^{3}$ Department of Clinical Science, Intervention and Technology, Division of Pediatrics, Karolinska Institutet, Stockholm, Sweden

\section{Correspondence to} Dr Lena Backström-Eriksson; lena.backstrom.eriksson@ki. se

\section{ABSTRACT}

Background: Cystic fibrosis (CF) is the most common autosomal recessive, life-shortening disease among people of European origin. Type of genetic mutation and regular physical exercise has an impact on clinical outcome. This cross-sectional study explores the associations between genetics, medical status, physical exercise and psychological well-being in adult patients with CF.

Methods: Adult patients with $\mathrm{CF}$ ( $\mathrm{N}=68$; mean age: 32.2; range 18-67 years; $46 \%$ women) completed the Cystic Fibrosis Questionnaire-Revised and Hospital Anxiety Depression Scale. Measures about lung function/forced expiratory volume in $1 \mathrm{~s}$ per cent predicted, body mass index, physical working capacity, immunoglobulin G, CF Transmembrane Conductance Regulator (CFTR) mutations, and physical exercise were obtained. structural equation modelling was used to fit models to data.

Results: A cftr gene mutation $\times a g e$ interaction effect indicates a psychological disadvantage increasing with age of having more severe CFTR mutations; $>65 \%$ of the effect is mediated by medical status. Physical exercise has a positive effect on psychological wellbeing, but $>75 \%$ of the effect is mediated by medical status.

Conclusions: Psychological well-being decreases with age in patients with more severe cftr mutations, to a large extent due to a parallel deterioration of medical status. Physical exercise has a positive effect on psychological well-being if resulting in better health only. To manage the complexity of these patients' needs, the CF-care should emphasise a holistic approach and offer individualised exercise/treatment programmes and psychological competence.

\section{INTRODUCTION}

Cystic fibrosis (CF) is the most common genetic life-shortening disease in populations of European origin. ${ }^{1}$ The disease is caused by mutations in the cftr gene and is affecting multiple organs such as the lungs, pancreas and liver. $\mathrm{CF}$ is a chronic disease and the

\section{KEY MESSAGES}

Does genetic mutation and physical exercise have associations with psychological well-being in adults with cystic fibrosis (CF)?

- Patients with CF homozygous or compound heterozygous for cftr gene mutation classes I and II with age get poorer psychological well-being with a parallel deterioration of health, and physical exercise contributes to psychological wellbeing in patients with CF only if resulting in improved health.

- The results will be helpful for the CF care identifying and strategically managing patients vulnerable for poor psychological well-being, a factor associated with poor adherence to treatment.

expected course of the disease is a progressive deterioration of health. The primary cause of mortality and morbidity in $\mathrm{CF}$ is progressive lung disease. Advances in the management of $\mathrm{CF}$ have led to dramatically improved prognosis and prolonged survival rate, ${ }^{2}$ and to a growing proportion of the patients with $\mathrm{CF}$ now being adults. However, the disease is still incurable and the CF treatment regimen is extraordinarily demanding for the patients, ${ }^{3}$ conditions that might have an impact on the well-being of adult patients with CF, health-related quality of life (HRQoL) and psychological health.

Concerning associations between HRQoL and medical status among adults and adolescents with CF, stability of quality of life (QoL) over time and an independence of QoL and lung function have been concluded. ${ }^{4}$ In contrast, another study showed that changes in clinical variables are associated with change in HRQoL over time. ${ }^{5}$ Similarly, positive associations between lung function and QoL have been found among adolescents and adults with $\mathrm{CF}^{6}{ }^{7} \mathrm{In}$ 
one study, adult patients with CF reported better QoL than people in general. ${ }^{7}$

Only a few studies explore the more complex relationship between QoL, psychological health (ie, anxiety and depression) and medical status in people with CF. Among adults and adolescents, it has been found that life satisfaction, ${ }^{8}$ a related aspect of QoL, was negatively associated with anxiety and depression and positively associated with lung function. ${ }^{8}$ Similar results were obtained in a study among adults. ${ }^{9}$ Also, associations between medical status and QoL as well as associations between QoL and anxiety and depression, respectively, have been found among adult patients with CF. ${ }^{10} 11$

Regular physical exercise can maintain and improve health in people with CF. ${ }^{12}$ However, only a few studies have explored associations between physical exercise and HRQoL in adult patients with CF. A substantially beneficial effect of exercise on QoL has been concluded in a recent review, ${ }^{13}$ and a recent study among adults and adolescents ${ }^{14}$ found positive associations between HRQoL and physical activity. In contrast, two studies evaluating home exercise programmes among adults and adolescents with CF did not show any improvement in total HRQoL. ${ }^{15}{ }^{16}$ On the other hand, in adult general populations a positive association between level of physical activity and HRQoL has been concluded. ${ }^{17}$

In non-somatically ill populations, it is known that physical exercise can improve anxiety and depression symptoms. ${ }^{18-20}$ No studies in patients with CF have explored the impact of physical exercise on anxiety and depression.

Cftr mutations have been grouped into different classes based on their predicted functional consequences for the CFTR protein, classes I-III being regarded as the most severe with an impact on the medical outcome..$^{21-24}$ The type of mutation possibly also has an impact on the patients' HRQoL and psychological health. Until now, no studies have explored this.

The aim of this study was to examine the associations between genetics, medical status, physical exercise and psychological well-being in Swedish adults with CF. It could be anticipated that patients with a 'more severe' mutation class would have a worse medical status and, as an association between medical status and psychological well-being can be expected, also a worse psychological well-being. Owing to the expected association, a mediation of the effect of mutation class on psychological well-being, via medical status, could also be predicted. For similar reasons, an effect of physical exercise on psychological well-being, mediated via medical status, could be anticipated.

\section{METHOD}

\section{Subjects}

Participants to the study were recruited from the Stockholm CF Center, the largest CF-center in Sweden. Patients of interest were adults ( $\geq 18$ years of age) with a confirmed diagnosis of $\mathrm{CF}$ and a known genotype.
Transplanted patients were excluded since the transplant changes the symptomatology, health status and treatment regimen dramatically. After exclusion, 91 participants remained as potential participants and of these 68 $(75 \%)$ volunteered to participate in the study; $46 \%$ women; age: 32.2, SD 11.1, range 18-67 years. Mean forced expiratory volume in $1 \mathrm{~s}\left(\mathrm{FEV}_{1}\right)$ percent predicted: 71.1, SD 28.1, range 22-125.

Of the potential participants, $25 \%$ did not participate in the study: $43 \%$ women; Mean age: 33, range 19-65, mean $\mathrm{FEV}_{1}$ percent predicted: 71.7, range 20.6-123.3.

The study was approved by the Central Ethical Review Board, Stockholm, ethical permission, nr 2007/1266-31.

\section{Procedures}

The patients were invited and informed about the study by regular mail, which was followed up by a phone call. If interested in participating, an appointment with the CF-team psychologist or social worker was scheduled. During this appointment, the patient, after completion of written informed consent/assent, completed CFQ-R and HADS. Completion of the questionnaires usually took $<20$ min. Patients who reported elevated HADS scores $(\geq 8)$ were followed up by the psychologist. After the appointment, a medical check-up was performed according to the routines for an outpatient clinic visit and $\mathrm{FEV}_{1}$ percent of predicted value and BMI were measured. Data collection was made consecutively between March 2008 and February 2009.

Data were completed with information from the national Swedish registry based on the latest annual check-up about immunoglobulin G (IgG), physical working capacity (PWC), amount of physical exercise and information about genotype.

\section{Measures}

Assessments of HRQoL should be patient centred and reflect the individual's subjective evaluation of his or her functioning and well-being. ${ }^{25}$ Since earlier associations have been found among adult patients with CF between HRQoL and anxiety and depression, respectively, ${ }^{10}{ }^{11}$ anxiety and depression were measured, and the measures were, together with the HRQoL measures, used as a proxy of 'psychological well-being'.

HRQoL was measured using the Swedish translation (submitted: Hochwälder J, Bergsten-Brucefors A, Hjelte L) of the teen/adult version of CFQ-R, a questionnaire developed to measure HRQoL specifically in people with $\mathrm{CF}^{25}$ The instrument consists of 50 items across 12 domains (table 1). Reply choices generally include ratings of frequency and difficulty on a scale graded 1-4 ( $1=$ 'always' to $4=$ 'never', and $1=$ 'a lot of difficulty' to 4 'no difficulty') or true/false ( $1=$ 'very true' to $4=$ =very false'). Some items have more pronounced response alternatives, for example, 'How do you assess your health right now?', 1='Excellent' to 4='Poor'). Scores are standardised to a range $0-100$ with higher scores indicating better HRQoL. Questions concerning demographic 
Table 1 Descriptive statistics separately for the four groups based on the official cftr gene mutation classification system

\begin{tabular}{|c|c|c|c|c|c|c|c|c|c|c|c|}
\hline & \multicolumn{2}{|c|}{ Group $1(n=8)$} & \multicolumn{2}{|c|}{ Group $2(n=30)$} & \multicolumn{2}{|c|}{ Group 3 (n=13) } & \multicolumn{2}{|c|}{ Group 4 (n=17) } & \multicolumn{2}{|c|}{ Total $(n=68)$} & \multirow[b]{2}{*}{$\mathbf{F} ¥$} \\
\hline & $\bar{M}$ & SD & $\overline{\mathbf{M}}$ & SD & $\overline{\mathbf{M}}$ & SD & $\overline{\mathbf{M}}$ & SD & $\bar{M}$ & SD & \\
\hline Age & 33.38 & 10.62 & 29.07 & 9.70 & 32.31 & 9.86 & 36.88 & 13.53 & 32.15 & 11.13 & 1.90 \\
\hline HADS A & 6.63 & 2.88 & 6.60 & 4.07 & 4.15 & 3.46 & 5.18 & 2.88 & 5.78 & 3.63 & 1.74 \\
\hline HADS D & 5.00 & 3.93 & 3.00 & 2.45 & 2.08 & 2.66 & 3.65 & 2.98 & 3.22 & 2.88 & 2.00 \\
\hline CFQ-R Tot & 69.74 & 13.69 & 76.11 & 15.25 & 72.03 & 19.23 & 78.25 & 14.85 & 75.10 & 15.73 & 0.73 \\
\hline $\mathrm{BMI}$ & 21.06 & 1.94 & 21.70 & 2.22 & 21.85 & 2.70 & 24.71 & 4.98 & 22.40 & 3.41 & $4.02^{*}$ \\
\hline $\mathrm{FEV}_{1}$ & 60.38 & 32.58 & 72.79 & 26.98 & 79.62 & 28.58 & 70.12 & 24.89 & 71.96 & 27.37 & 0.85 \\
\hline Phys exer & 2.29 & 2.51 & 4.27 & 6.68 & 1.19 & 1.90 & 1.47 & 1.88 & 2.73 & 4.82 & 0.64 \\
\hline $\lg G$ & 10.76 & 3.15 & 11.47 & 3.61 & 11.09 & 2.47 & 11.13 & 2.68 & 11.23 & 3.06 & 0.12 \\
\hline Work cap & 201.86 & 73.95 & 141.71 & 89.81 & 200.62 & 54.60 & 143.63 & 81.36 & 162.00 & 82.25 & $2.42 \dagger$ \\
\hline
\end{tabular}

${ }^{*} \mathrm{p}<0.05 ; \mathrm{p} \mathrm{p}<0.10$.

$\ddagger$ With $\mathrm{df}=3$ and 64 .

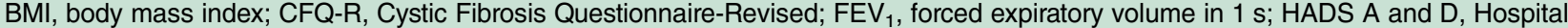

Anxiety and Depression Scale Anxiety and Depression; IgG, immunoglobulin G.

factors (age, sex, civil status, educational level, work/ school situation) are also answered. The questionnaire is until now translated into 34 languages and shows robust

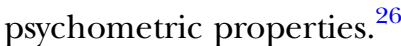

Anxiety and depression were measured using the Swedish validated version ${ }^{27}$ of Hospital Anxiety and Depression Scale (HADS), ${ }^{28}$ a well-validated ${ }^{29}$ screening tool developed to identify cases of anxiety and depression in somatic populations. The questionnaire has two subscales, anxiety and depression, and consists of 14 items graded 0-3. Anxiety and depression scores are obtained by summing up the scores of each subscale, yielding values $0-21$. Each subscale has three ranges: 0-7 (non-cases), 8-10 (mild-moderate anxiety or depression) and 11-21 (moderate-severe anxiety or depression). The cut off-scores are defined on the basis of psychiatric ranges of anxiety and depression disorders.

Four measures were used as proxies of medical status: $\mathrm{FEV}_{1} \%$ predicted, BMI, IgG and PWC. Annual checkups were performed according to European guidelines. $^{30}$ Information about pulmonary function, for example, $\mathrm{FEV}_{1} \%$ predicted (Solymar and Hedenström reference equations for patients $<19$ and $\geq 19$ years, respectively), ${ }^{31-33} \mathrm{BMI}$ (weight $\left.(\mathrm{kg}) /(\text { height }(\mathrm{m}))^{2}\right)$, IgG (routine in-house methods of protein electrophoresis), PWC (watt) (performed on an electrically braked bicycle ergometer) and amount of physical exercise (hours per week) was retrieved from medical records and the national Swedish CF-register. As high values on IgG indicate low degree of medical status high values were reversed to low and low values to high in order to get a variable were, similarly as on pulmonary function, BMI and PWC, high values indicate a high degree of medical status. These data were taken from the annual check-up performed during the period March 2008 to February 2009. In a few cases, since data were not available from the annual check-up during the present period, corresponding data were taken from the annual check-up performed in the year closest before or closest after.
Information regarding the patients' $c f t r$ gene mutation was collected from the Swedish National CF-register. The patients were divided into groups based on the official CFTR-database classification system: ${ }^{34}$

Group 1. Homozygotes: Class I

Group 2. Homozygotes: Class II

Group 3. Compound heterozygotes: Classes I and II

Group 4. Heterozygotes: Class I + 'other' and Class II

+ 'other', where 'other' corresponds to Classes III, IV,

$\mathrm{V}$ and VI. Furthermore, this group also contained patients homozygous or heterozygous for classes III-VI.

Since the mutation Classes I and II are causing a more severe disease, ${ }^{21} 35$ they are put together in the statistical analyses as 'Group 1-3' and compared to 'Group 4'.

\section{Statistical analyses}

Structural equation modelling (SEM) can be used to analyse associations between latent variables, which are unobserved constructs measured through manifest (observed) indicators. By having multiple indicators, one can expect to get a more complete and reliable measurement of the latent construct of interest compared with that provided by a single indicator. SEM could be described as a combination of factor and regression analysis. However, differently from traditional regression analysis, SEM does not assume that independent variables are measured without measurement error, an approach that tends to give more realistic parameter estimates. ${ }^{36}$

SEM was used to fit two different models to the data. It should be noted that these two models are about two different questions, one about the effect of $c f t r$ mutation class and the other about the effect of exercise, rather than being two competing models trying to answer the same question. In both models, medical status was defined as a latent variable with the four manifest indicators (proxies) lung function, BMI, PWC and IgG (reversed). The correlations between lung function, PWC and IgG were high (>0.55), while the correlation between these three and BMI was quite low (0.15-0.29). BMI was still kept as an indicator of medical status 
because it is often used as such among patients with CF. The Cronbach's $\alpha$ of these four measures was 0.73 , indicating satisfactory homogeneity.

In model 1, Groups 1-3, age and Groups 1-3×age interaction were used as predictors of 16 different aspects reflecting psychological well-being, and with medical status as a mediator, mediating the effect of these predictors on the different aspects of psychological well-being. Effects were adjusted for the patient's sex, having a partner or not and educational level. Model 1 is illustrated in figure 1 with the CFQ-R total score as the outcome.

In model 2, level of exercise was used as the sole independent variable instead of Groups 1-3, age and Groups 1-3×age interaction. In this model, Groups 1-3 and age

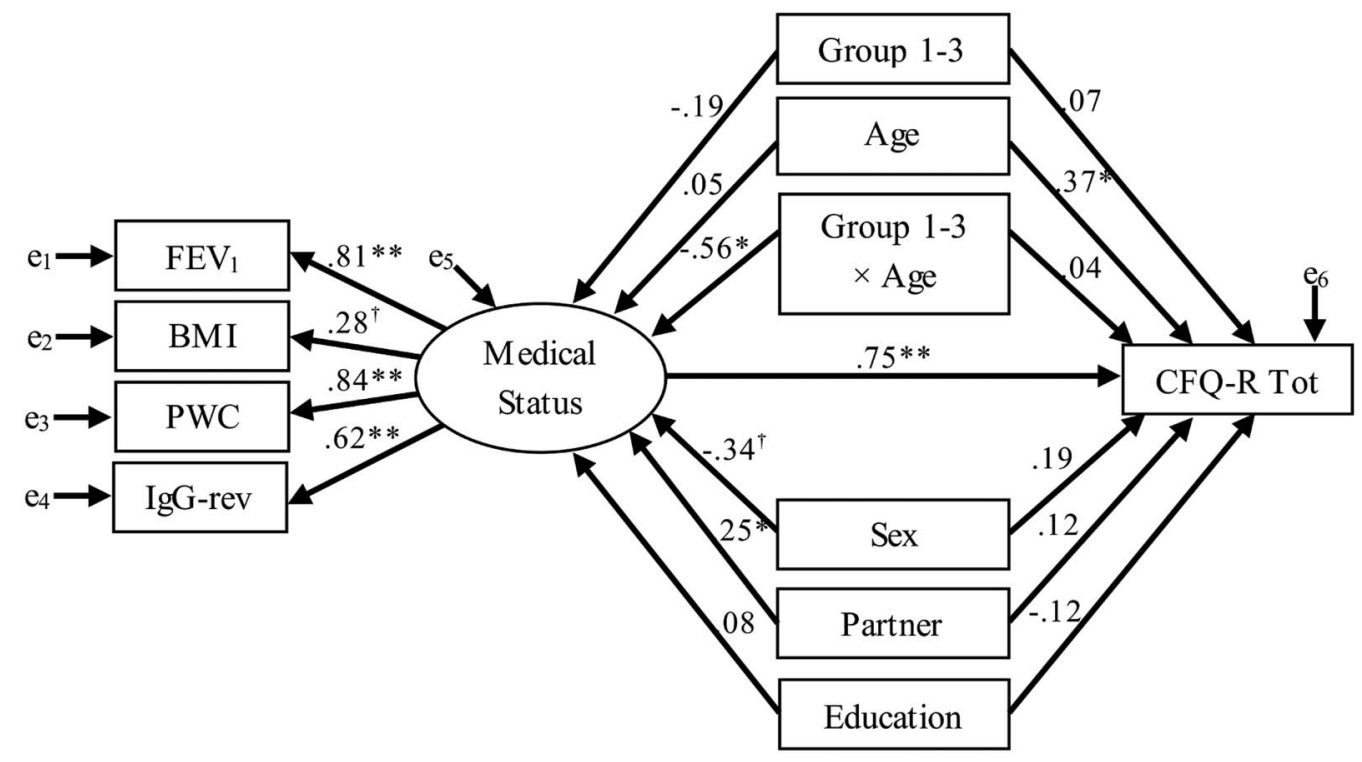

Figure 1 Illustration of model 1, with CFQ-R total score as the outcome. The manifest (=observed) variables FEV 1 , BMI, PWC and IgG (reversed) are used as indicators of the latent variable medical status, and this latent variable is included as a mediator mediating the effects of the predictors Groups 1-3, age and the Groups 1-3xage interaction effect on CFQ-R total score. The effects are adjusted for the effects of sex, having a partner or not and level of education. The parameter values are standardised regression coefficients. $\dagger p<0.10,{ }^{\star} p<0.05,{ }^{* *} p<0.001$. BMI, body mass index; CFQ-R, Cystic Fibrosis Questionnaire-Revised; $\mathrm{FEV}_{1}$, forced expiratory volume in $1 \mathrm{~s}$; IgG, immunoglobulin G; PWC, physical working capacity.

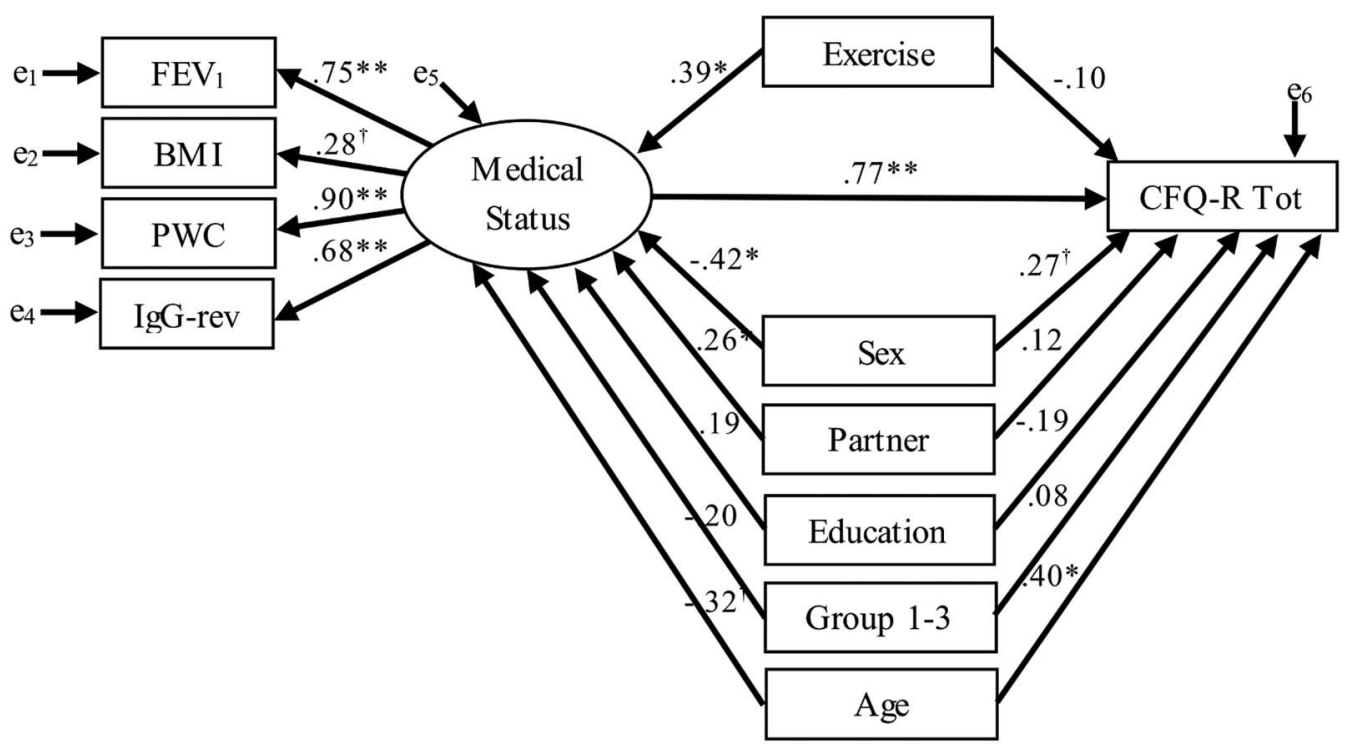

Figure 2 Illustration of model 2, with CFQ-R total score as the outcome. The manifest (=observed) variables FEV 1 , BMI, PWC and $\operatorname{lgG}$ (reversed) are used as indicators of the latent variable medical status, and this latent variable is included as a mediator mediating the effect of amount of physical exercise on CFQ-R total score. The effects are adjusted for the effects of sex, having a partner or not, level of education, groups $1-3$ and age. The parameter values are standardised regression coefficients. $t p<0.10,{ }^{*} p<0.05,{ }^{* *} p<0.001$. BMI, body mass index; CFQ-R, Cystic Fibrosis Questionnaire-Revised; $F E V_{1}$, forced expiratory volume in $1 \mathrm{~s}$; IgG, immunoglobulin G; PWC, physical working capacity. 
were adjusted for together with the patient's sex, having a partner or not and educational level. Model 2 is illustrated in figure 2 with the CFQ-R total score as the outcome. Analyses were conducted with Mplus V.7.3 software using Maximum Likelihood with Robust SEs estimation (Muthén and Muthén, 1998-2012).

\section{RESULTS}

Characteristics of the sample are presented in table 1 and online supplementary material S1. In almost all comparisons between the groups, the differences are not significant. However, the results are generally poorer for Group I. Levels of anxiety and depression for the total sample are in line with previous findings in a Swedish adult CF population, only marginally different compared to Swedish normative data. ${ }^{37}$

The direct, indirect/mediated and total effects of medical status, Groups 1-3, age and Groups 1-3×age interaction (model 1), and of medical status together with level of exercise (model 2) on the four different outcomes reflecting the aspects of the variable psychological well-being, are presented in table 2. The same effects on the CFQ-R subscales are presented in online supplementary table S2.

For all outcomes reflecting aspects of the variable psychological well-being, except the CFQ-R Digest subscale and HADS-Anxiety, the Groups 1-3×age interaction effect indicates that the disadvantage of homozygosity, or compound heterozygosity, for Classes I and II increases with age and in seven cases this total interaction effect is significant. Leaving the CFQ-R Digest subscale aside, when the Groups 1-3×age interaction effect is significant, a substantial $(>65 \%)$ portion of the effect is mediated via medical status. This means that the increase with age in the psychological disadvantage of homozygosity, or compound heterozygosity, for Classes I and II is to a large extent accounted for by a parallel increase in a medical disadvantage of homozygosity, or compound heterozygosity, for Classes I and II (figure 3). For the CFQ-R subscales Emotion, Treat, Health, Weight and Respiration, a positive direct effect and a negative indirect effect cancel each other out resulting in a non-significant total Groups 1-3×age interaction effect (see online supplementary table S2).

To summarise: The psychological disadvantage of having the mutation classes causing a more severe disease increase with age, and a large portion of this increase in disadvantage is accounted for by a matching deterioration in medical status.

Physical exercise has a positive total effect on psychological well-being, except when measured with the CFQ-R Emotion subscale or HADS-Anxiety, and in eight instances this total effect is significant. In these eight cases, more than $75 \%$ of the effect is mediated via medical status. It is interesting to note that in no case is the direct effect of exercise significant, and in most cases it is even slightly negative. This may indicate that

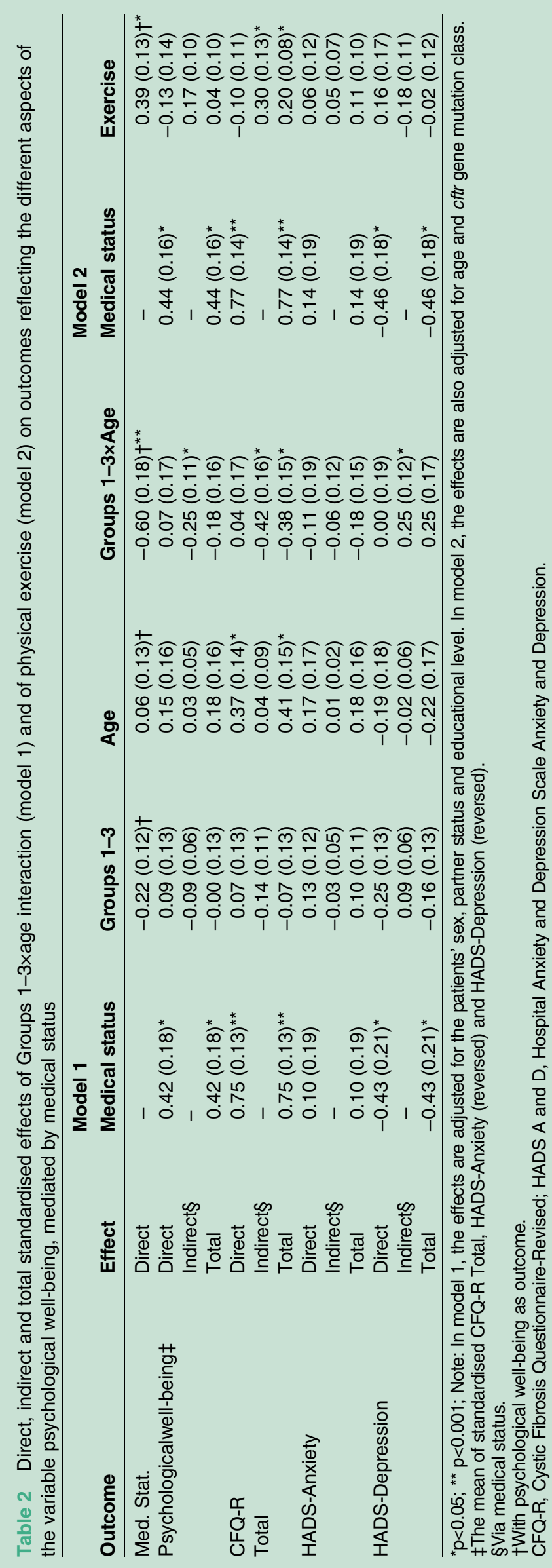


Figure 3 The association between age and CFQ-R total score (left) and medical status (standardised to mean zero and SD one) (right) for patients homozygous or compound heterozygous for Classes I and II (filled circles and solid lines) and those not having these mutation classes (open circles and dotted lines). CFQ-R, Cystic Fibrosis Questionnaire-Revised.
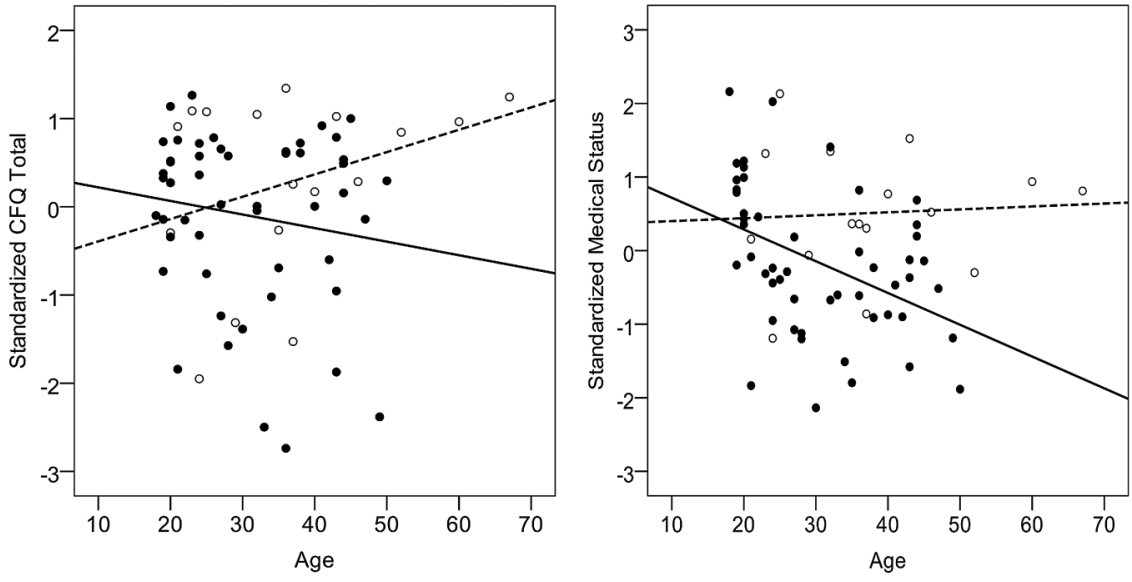

there would be no advantage of physical exercise on psychological well-being if not accompanied by a positive effect on medical status.

\section{DISCUSSION}

It has been concluded earlier that there is no elevated risk for anxiety and depression in the Swedish adult CF-population compared to the general population. ${ }^{37}$ The present findings are in accordance with those from earlier studies that have found an association between type of $c f t r$ gene mutation and medical outcomes ${ }^{2135}$ as well as between lung function and psychological wellbeing among patients with $\mathrm{CF}^{5}{ }^{8-11}$ Above this, the present study indicates that the type of $c f t r$ gene mutation has an indirect/mediated effect on psychological well-being via medical status. Our results indicate that psychological well-being decreases with age for patients homozygous, or compound heterozygous, for Classes I and II. However, this decrease is to a large extent accounted for by a deterioration in health; thus, the type of $c f t r$ gene mutation cannot be said to have a direct impact on psychological well-being.

Our results further indicate that physical exercise has a positive effect on psychological well-being only if accompanied by a positive association with medical status. In no case is the direct effect of exercise significant, and in most cases it is even slightly negative. This may indicate that there would be no advantage of physical exercise on psychological well-being if not accompanied by a positive effect on health. However, neither the outcome 'anxiety' nor 'emotion' was included in the effect, and this statement therefore needs further research. Similar to two studies, ${ }^{13} 14$ but contrary to two others, ${ }^{1516}$ our results show that physical exercise is associated with higher HRQoL. Our findings are maybe not comparable with the results from studies evaluating relatively short exercise/treatment programmes in patients who have not been exercising regularly before the study, and in which effects on lung function are not achieved. ${ }^{15} 16$ It should be taken into account that physical exercise is an essential part of the Swedish treatment regimen since the 1980 s and prescribed from the day of diagnosis. Since early childhood, a large part of the present sample has been exercising regularly and had their daily airway clearance partly based on exercise.

The negative (decreasing) effect of physical exercise on anxiety and depression symptoms, seen in nonsomatic populations, ${ }^{18-20}$ is not seen in adults with CF. However, in these studies, medical status is not taken into account and it is therefore unknown if and how the medical status influences the effects of exercise on psychological well-being. Furthermore, physical exercise has not been tested earlier as an intervention for depressed or anxious non-exercising patients with CF; exercise may decrease depression and anxiety symptoms.

Since this is the first study including genetics, medical status and amount of physical exercise in the examination of psychological well-being in adult patients with CF, further research is needed to better understand the complexity of the associations found.

Both ageing patients homozygous, or compound heterozygous, for Classes I and II, and patients struggling with a high amount of exercise without improving their health should be seen as vulnerable for psychological distress. Since poor psychological well-being has a negative impact on adherence to treatment, ${ }^{38}$ these issues are extra important to address. The CF care should take the complexity of these associations into account, emphasising a broad, holistic approach. To balance the need for a high amount of exercise, individualised care receptive for the patients' needs, focusing on pleasurable alternative exercise and treatment forms, should be offered. Patients should be supported to find aspects of QoL that can be improved and maintained. Regular measurement of HRQoL and, according to international guidelines, $^{39}$ screening for symptoms of psychological distress should be offered.

Furthermore, the CF-centre staff should be trained in psychological skills to address and meet these patients' needs. According to European guidelines, ${ }^{40}$ psychologists should be integrated in the CF-care offering assessment and evidence-based interventions suitable for people dealing with stressful conditions. 
Despite the good representativeness of the present CF-centre, this study has some limitations. First, the sample size and the groups are small; it might be interesting for the larger CF-centres or countries with larger CF-populations to further investigate these findings. However, we would like to emphasise that the effects found were very similar independent of which measure of psychological well-being was used, and this could be seen to indicate that the results are quite stable and not due to some random coincidence. Furthermore, the amount of physical exercise and some of the medical data were not measured at the same clinical visit as the medical parameters collected in connection to completion of the questionnaires. Physical exercise was reported directly to the physiotherapists, which possibly could have influenced the patient to overestimate their amount of exercise. However, this would affect the present findings only if degree of overestimation correlates with medical status or psychological well-being. The design of this study was cross-sectional; a longitudinal design should have given more information about changes over time and their predictors. Finally, although HADS is well validated and developed for use in somatic populations, a recent study ${ }^{41}$ revealed that limitations are found in the HADS Depression subscale. However, the main focus of this study was not levels or prevalence of depression.

\section{CONCLUSIONS}

Patients homozygous, or compound heterozygous, for Classes I and II with age get poorer psychological wellbeing with a parallel deterioration of health. Furthermore, physical exercise has a positive effect on psychological well-being only if associated with better health. To manage the complexity of these associations, the CF-care should emphasise a holistic approach and offer individualised exercise/treatment programmes and psychological competence.

Acknowledgements The authors thank all the patients who participated.

Contributors LB-E planning of the study concept, data collection, statistical analysis and interpretation of the data, drafting of the article. AB-B initial planning of the study, data collection, critically reviewing of the article. LH initial planning of the study, critically reviewing of the article, drafting of the article. BM planning of the study concept, drafting/critically reviewing of the article. KS planning of the study concept, statistical analysis and interpretation of the data, drafting/critically reviewing of the article, PI. All authors approved the final version published, and agree to be accountable for all aspects of the work in ensuring that questions related to the accuracy or integrity of any part of the work are appropriately investigated and resolved.

Funding The study was funded from Swedish Cystic Fibrosis Foundation (Riksförbundet Cystisk Fibros, RfCF), and Division of Psychology, Karolinska University Hospital is gratefully acknowledged.

Competing interests None declared.

Ethics approval Central Ethical Review Board, Stockholm, ethical permission, nr 2007/1266-31.

Provenance and peer review Not commissioned; externally peer reviewed.

Data sharing statement No additional data are available.
Open Access This is an Open Access article distributed in accordance with the Creative Commons Attribution Non Commercial (CC BY-NC 4.0) license, which permits others to distribute, remix, adapt, build upon this work noncommercially, and license their derivative works on different terms, provided the original work is properly cited and the use is non-commercial. See: http:// creativecommons.org/licenses/by-nc/4.0/

\section{REFERENCES}

1. FitzSimmons SC. The changing epidemiology of cystic fibrosis. J Pediatr 1993;122:1-9.

2. Davis PB. Cystic fibrosis since 1938. Am J Respir Crit Care Med 2006;173:475-82.

3. Sawicki GS, Sellers DE, Robinson WM. High treatment burden in adults with cystic fibrosis: challenges to disease self-management. J Cyst Fibros 2009;8:91-6.

4. Goldbeck L, Zerrer S, Schmitz TG. Monitoring quality of life in outpatients with cystic fibrosis: feasibility and longitudinal results. J Cyst Fibros 2007;6:171-8.

5. Abbott J, Morton AM, Hurley MA, et al. Longitudinal impact of demographic and clinical variables on health-related quality of life in cystic fibrosis. BMJ Open 2015;5:e007418.

6. Gee L, Abbott J, Hart A, et al. Associations between clinical variables and quality of life in adults with cystic fibrosis. J Cyst Fibros 2005;4:59-66.

7. Wahl AK, Rustøen T, Hanestad BR, et al. Living with cystic fibrosis: impact on global quality of life. Heart Lung 2005;34:324-31.

8. Besier T, Goldbeck L. Growing up with cystic fibrosis: achievement, life satisfaction, and mental health. Qual Life Res 2012;21:1829-35.

9. Riekert KA, Bartlett SJ, Boyle MP, et al. The association between depression, lung function, and health-related quality of life among adults with cystic fibrosis. Chest 2007;132:231-7.

10. Yohannes AM, Willgoss TG, Fatoye FA, et al. Relationship between anxiety, depression, and quality of life in adult patients with cystic fibrosis. Respir Care 2012;57:550-6.

11. Havermans T, Colpaert K, Dupont LJ. Quality of life in patients with cystic fibrosis: association with anxiety and depression. J Cyst Fibros 2008;7:581-4.

12. Smyth AR, Bell SC, Bojcin S, et al. European Cystic Fibrosis Society Standards of Care: best practice guidelines. J Cyst Fibros 2014;13 (Suppl 1):S23-42.

13. Dwyer TJ, Elkins MR, Bye PT. The role of exercise in maintaining health in cystic fibrosis. Curr Opin Pulm Med 2011;17:455-60.

14. Hebestreit $\mathrm{H}$, Schmid K, Kieser S, et al. Quality of life is associated with physical activity and fitness in cystic fibrosis. BMC Pulm Med 2014;14:9.

15. Schmidt AM, Jacobsen U, Bregnballe V, et al. Exercise and quality of life in patients with cystic fibrosis: a 12-week intervention study. Physiother Theory Pract 2011;27:548-56.

16. Rovedder PM, Flores J, Ziegler B, et al. Exercise programme in patients with cystic fibrosis: a randomized controlled trial. Respir Med 2014:108:1134-40.

17. Bize R, Johnson JA, Plotnikoff RC. Physical activity level and health-related quality of life in the general adult population: a systematic review. Prev Med 2007;45:401-15.

18. Martinsen EW. Physical activity in the prevention and treatment of anxiety and depression. Nord J Psychiatry 2008;62(Suppl 47):25-9.

19. Asmundson GJ, Fetzner MG, Deboer LB, et al. Let's get physical: a contemporary review of the anxiolytic effects of exercise for anxiety and its disorders. Depress Anxiety 2013;30:362-73.

20. Josefsson T, Lindwall M, Archer T. Physical exercise intervention in depressive disorders: meta-analysis and systematic review. Scand J Med Sci Sports 2014;24:259-72.

21. Kerem B, Kerem E. The molecular basis for disease variability in cystic fibrosis. Eur J Hum Genet 1996;4:65-73.

22. Zielenski J. Genotype and phenotype in cystic fibrosis. Respiration 2000;67:117-33.

23. Zielenski J, Tsui LC. Cystic fibrosis: genotypic and phenotypic variations. Annu Rev Genet 1995;29:777-807.

24. Welsh MJ, Smith AE. Molecular mechanisms of CFTR chloride channel dysfunction in cystic fibrosis. Cell 1993;73:1251-4.

25. Quittner AL, Buu A, Messer MA, et al. Development and validation of the Cystic Fibrosis Questionnaire in the United States: a health-related quality-of-life measure for cystic fibrosis. Chest 2005;128:2347-54.

26. Quittner AL, Sawicki GS, McMullen A, et al. Psychometric evaluation of the Cystic Fibrosis Questionnaire-Revised in a national sample. Qual Life Res 2012;21:1267-78.

27. Lisspers J, Nygren A, Söderman E. Hospital Anxiety and Depression Scale (HAD): some psychometric data for a Swedish sample. Acta Psychiatr Scand 1997;96:281-6. 
28. Zigmond AS, Snaith RP. The hospital anxiety and depression scale. Acta Psychiatr Scand 1983;67:361-70.

29. Bjelland I, Dahl AA, Haug TT, et al. The validity of the Hospital Anxiety and Depression Scale. An updated literature review. J Psychosom Res 2002;52:69-77.

30. Kerem E, Conway S, Elborn S, et al. Standards of care for patients with cystic fibrosis: a European consensus. J Cyst Fibros 2005;4:7-26.

31. Solymar L, Aronsson PH, Bake B, et al. Nitrogen single breath test, flow-volume curves and spirometry in healthy children, 7-18 years of age. Eur J Respir Dis 1980;61:275-86.

32. Hedenstrom H, Malmberg P, Agarwal K. Reference values for lung function tests in females. Regression equations with smoking variables. Bull Eur Physiopathol Respir 1985;21:551-7.

33. Hedenstrom H, Malmberg P, Fridriksson HV. Reference values for lung function tests in men: regression equations with smoking variables. Ups J Med Sci 1986;91:299-310.

34. Clinical and Functional Translation of CFTR website. http://www. cftr2.org, entered 17 March 2016.

35. Geborek A, Hjelte L. Association between genotype and pulmonary phenotype in cystic fibrosis patients with severe mutations. J Cyst Fibros 2011;10:187-92.
36. Raykov T, Marcoulides GA. A first course in structural equation modeling. 2nd edn. Mahvah, NJ: Lawrence Erlbaum Associates, 2006.

37. Backstrom-Eriksson L, Sorjonen K, Bergsten-Brucefors A, et al. Anxiety and depression in adults with cystic fibrosis: a comparison between patients and the general population in Sweden and three other European countries. BMC Pulm Med 2015;15:121.

38. Cruz I, Marciel KK, Quittner AL, et al. Anxiety and depression in cystic fibrosis. Semin Respir Crit Care Med 2009;30:569-78.

39. Quittner AL, Abbott J, Georgiopoulos AM, et al. International Committee on Mental Health in Cystic Fibrosis: Cystic Fibrosis Foundation and European Cystic Fibrosis Society consensus statements for screening and treating depression and anxiety. Thorax 2016;71:26-34.

40. Conway S, Balfour-Lynn Intramuscular, De Rijcke K, et al. European Cystic Fibrosis Society Standards of Care: Framework for the Cystic Fibrosis Centre. J Cyst Fibros 2014;13(Suppl 1):S3-S22.

41. Quittner AL, Goldbeck L, Abbott J, et al. Prevalence of depression and anxiety in patients with cystic fibrosis and parent caregivers: results of the International Depression Epidemiological Study across nine countries. Thorax 2014:69:1090-7. 